\title{
Methemoglobinemia due to topical pharyngeal anesthesia during endoscopic procedures
}

\author{
This article was published in the following Dove Press journal: \\ Local and Regional Anesthesia \\ 29 November 2010 \\ Number of times this article has been viewed
}

\section{Srikanth Vallurupalli \\ Southern Illinois University College of Medicine, Springfield, IL, USA}

Correspondence: Srikanth Vallurupalli Southern Illinois University College of Medicine, 70I N First Street, PO Box 19636, Springfield,

IL 62794, USA

Tel + | 21754569| 4

Email svallurupalli@siumed.edu

\begin{abstract}
Acquired methemoglobinemia is a known complication of topical anesthesia during endoscopic procedures. Benzocaine has been implicated in a majority of cases and has been removed from some hospital formularies. However, a lot of uncertainty exists about the risk factors for this uncommon complication. This review attempts to summarize the current knowledge of this condition and describe known risk factors. Anesthetic and patient-specific risk factors are described along with some hypothetical procedure-related factors. Clinicians should identify patients who are at high risk and apply appropriate postprocedure screening. Prospective studies are needed to further our knowledge and improve patient safety.
\end{abstract}

Keywords: acquired methemoglobinemia, topical anesthetics, oxidative stress, endoscopy, transesophageal echocardiography (TEE)

\section{Introduction}

Endoscopic intubation of the oropharyngeal mucosa is commonly employed in the diagnosis and treatment of various disorders. Millions of such endoscopic procedures are performed every year around the world. Complications are infrequent and are related either to the procedure or the anesthesia provided to facilitate it. Topical anesthetics are widely used during endoscopy. Sprays are used to anesthetize the mucosa and suppress gag reflex, the tip of the endoscope is often lubricated with an anesthetic gel, and an anesthetic solution is sometimes instilled, especially in the bronchial mucosa. Methemoglobinemia is a rare complication of the use of topical anesthetics for mucosal anesthesia. It results from excess concentration of methemoglobin where the iron in the heme portion of the hemoglobin molecule remains in its ferric state. ${ }^{1}$ It has been reported with use of both benzocaine and lidocaine which are commonly used in this setting. The purpose of this review is to summarize our current knowledge of the etiopathogenesis of methemoglobinemia due to topical anesthetics in the setting of endoscopy.

\section{Scope of the problem}

The exact incidence of topical anesthesia-induced acquired methemoglobinemia during endoscopy is unknown. There have been no prospective studies to date. Based on retrospective series, reported incidence with benzocaine varies from $0.067 \%$ to $0.45 \%$ during transesophageal echocardiography (TEE) ${ }^{2-4}$ and $0.014 \%$ during bronchoscopy. ${ }^{5}$ Topical anesthesia for endoscopic procedures was responsible for 3\% of all cases of acquired methemoglobinemia encountered at a tertiary care hospital. ${ }^{6}$ Moore and colleagues identified 132 cases of definite or probable methemoglobinemia due to 
benzocaine reported to the United States Food and Drug Administration's Federal Drug Adverse Event Reporting System between November 1997 and March 2002. ${ }^{7}$ Though the incidence during endoscopy is not reported, 93\% of these cases were associated with the spray formulation which is typically used for endoscopy. Benzocaine was also the most common offending agent noted in an excellent review of the existing literature by Guay. ${ }^{8}$ Based on these data, several institutions including the Veterans Administration have removed benzocaine from their formulary. ${ }^{9}$

There are many barriers to recognizing the true scope of the problem. Patients may remain asymptomatic with significant levels of methemoglobin. Even patients with symptoms may be misdiagnosed due to the nonspecific nature of the complaints, the physician's lack of knowledge of the disorder, and the absence of routine monitoring. Pulse oximetry, which is commonly employed in monitoring patients undergoing endoscopy, is unreliable for the diagnosis of methemoglobinemia. Thus, it is safe to assume that the true incidence is much higher.

\section{Pathophysiology}

Oxidation of the iron molecule from its ferrous to ferric state leads to the formation of methemoglobin. ${ }^{1,10}$ Methemoglobin is constantly produced and reduced in the body (Figure 1). Production of methemoglobin occurs constantly in the body due to three mechanisms:

1. Auto-oxidation: When molecular oxygen binds to deoxyhemoglobin, one electron is partially transferred from heme iron $\left(\mathrm{Fe}^{+2}\right)$ to the bound oxygen, forming a ferric-superoxide anion. During deoxygenation, most of the oxygen leaves deoxyhemoglobin as molecular oxygen, but a small amount departs as a superoxide radical. This process results in molecules in which the heme iron remains in its ferric $\left(\mathrm{Fe}^{+3}\right)$ state. This process occurs spontaneously at a rate of about $1 \%$.

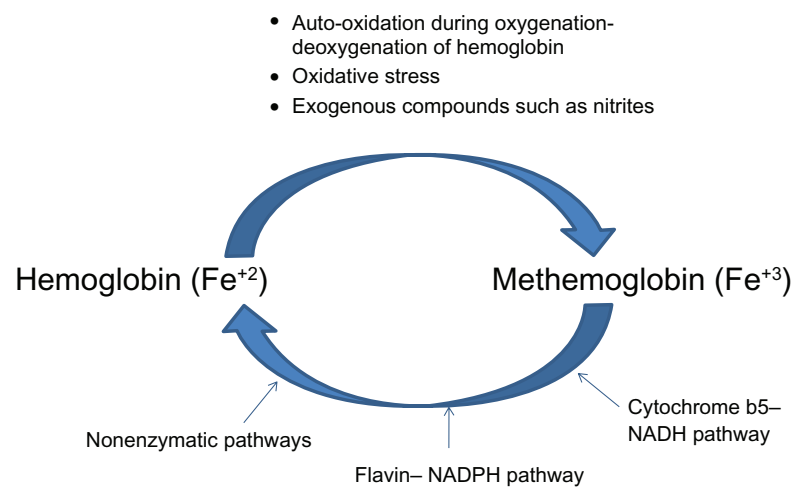

Figure I Methemoglobin levels are tightly controlled in the human body by an intricate balance between mechanisms that produce and reduce methemoglobin.
2. Oxidative stress: Free radicals and other endogenous compounds such as superoxide, nitric oxide, and hydrogen peroxide are produced during metabolic activity in the body. These molecules lead to oxidation of the ferrous moiety in heme, leading to methemoglobin formation.

3. Exogenous causes: Ingestion of exogenous compounds such as nitrites that may be present in food and water can result in production of compounds such as superoxide radicals.

Overall, a combination of these factors results in the production of a small amount of methemoglobin every day under normal conditions. At least three different mechanisms exist in the human body to effectively reduce methemoglobin:

1. Cytochrome b5-NADH pathway: This pathway consists of a redox cycle which consists of cytochrome b5 (cytb5) and its reductase (cytb5R). Cytb5R contains an FAD (flavin adenine dinucleotide) group which accepts electrons from NADH (nicotinamide adenine dinucleotide), gets reduced to FADH2 (dihydro FAD) which in turn reduces cytb5. Reduced cytb5 donates electrons to the methemoglobin molecule and returns the iron to its ferrous state. ${ }^{10}$

2. Flavin-nicotinamide adenine dinucleotide phosphate (NADPH) pathway: This is mediated via flavin methemoglobin reductase which relies on NADPH as a source of electrons. Under physiological conditions, there are no electron carriers which can interact with NADPH, so the importance of this pathway under normal conditions is unknown. Activation of this pathway occurs in the presence of methylene blue or riboflavin which serve as electron carriers.

3. Nonenzymatic pathways: Direct reduction of methemoglobin to hemoglobin is mediated by various substances such as ascorbic acid, reduced glutathione, etc. ${ }^{10}$

Of these, the cytochrome b5-NADH pathway accounts for about $95 \%$ of erythrocyte-reducing capacity and is considered the only physiologically important pathway. However, a recent study questions the relative importance of each of these pathways. ${ }^{11}$ Under normal physiological conditions, the pathways that produce and reduce methemoglobin maintain a methemoglobin level of $1 \%-2 \%$ and prevent toxicity.

Methemoglobinemia occurs when these pathways are overwhelmed by excessive methemoglobin production usually in the setting of increased oxidative stress. High concentrations of methemoglobin cause functional anemia by two mechanisms: by impairing the oxygen carrying capacity of the blood and by shifting the oxygen dissociation curve to the left resulting in tissue hypoxia due to impaired oxygen 
release. In the setting of endoscopy, methemoglobinemia appears to occur in a subset of patients who appear to have a combination of anesthetic-, patient-, and procedure-related factors.

\section{Anesthetic-specific factors}

Topical anesthetics work by blocking voltage gated sodium channels on neurons. ${ }^{12}$ They are broadly classified into two groups based on their chemical structure: esters and amides. The most commonly used topical anesthetics during endoscopy in the United States are benzocaine (ester) and lidocaine (amide). There are relatively few cases of methemoglobinemia reported with lidocaine use which is surprising considering that it is widely used. ${ }^{8}$ The exact mechanism involved or the metabolites have not been described. As noted above, most cases of acquired methemoglobinemia in the medical literature have been reported with benzocaine use. Benzocaine is metabolized by plasma pseudocholinesterase to ethanol and para aminobenzoic acid. Animal studies describe differences in metabolism of benzocaine which may sometimes result in the production of a toxic metabolite (an aniline-containing N-hydroxyl derivative) capable of inducing methemoglobinemia. ${ }^{13}$ Similar differences in metabolism may exist in humans which explains why only a few individuals who are exposed to benzocaine develop methemoglobinemia. Guertler et al demonstrated that a 2 -second spray of $20 \%$ benzocaine spray causes a statistically significant increase in serum methemoglobin levels at 20, 40, and 60 minutes after application from baseline $(0.8 \%$ vs $0.9 \%, P<0.05)$ in a group of normal volunteers and those undergoing upper gastrointestinal endoscopy. ${ }^{14}$

The dose of anesthetic administered can also play an important role in the pathogenesis. Excess amount of drug can result in increased production of toxic metabolites. A dose of $15 \mathrm{mg} / \mathrm{kg}$ in infants and between 150 and $300 \mathrm{mg}$ of benzocaine have been implicated. ${ }^{15}$ Multiple reports have described the association between inappropriately high doses of benzocaine (due to excessive number of or increased duration of sprays) and the occurrence of methemoglobinemia. Two major nonmetered formulations of benzocaine spray are widely used: $14 \%$ benzocaine (Cetacaine ${ }^{\circledR}[$ which contains butamben and tetracaine]; Cetylite Industries, Pennshauken, NJ) and 20\% benzocaine (Hurricane spray and other formulations). The instructions for use indicate that physicians adhere to a specific amount of spraying time and avoid multiple sprays. The difficulty with following these dosage instructions has been previously described. The instructions involve using a limited number of sprays which should be administered within a specific duration. With nonmetered canisters, it is not possible to precisely control spraying time. Nonmetered canisters also deliver differing doses based on canister orientation and the residual volume. ${ }^{16}$ A metered dose benzocaine canister (Topex ${ }^{\circledR}$; Sultan Healthcare, Hackensack, NJ) is now available and may help deliver precise doses. It is important to remember that cases of methemoglobinemia have been described even with the administration of recommended doses. ${ }^{7}$ Finally, the presence of mucosal trauma either from pre-existing tubes or due to endoscopy itself may cause increased absorption of the drug.

\section{Patient-specific factors}

Several patient-specific risk factors have been described. It should be noted that these factors have been best studied with the use of benzocaine.

\section{Age}

Extremes of age (infants and the elderly) are predisposed to acquired methemoglobinemia. Neonates have lower levels of functional cytb5R. Hemoglobin $\mathrm{F}$ is also more easily oxidized than hemoglobin A. ${ }^{17,18}$ The functional activity of cytb5R decreases with age. In addition, the elderly are much more likely to have co-existing medical conditions and use medications such as nitrates that may predispose to methemoglobinemia.

\section{Anemia}

The presence of anemia has been noted to be significantly higher in patients who develop methemoglobinemia than controls $(88.4 \%$ vs $44.7 \% ; P=0.002){ }^{2}$ The presence of a low baseline hemoglobin level makes patients more likely to exhibit signs of the functional anemia induced by increased methemoglobin. The cause of anemia may also predispose to methemoglobinemia. Patients who have anemia of chronic disease (due to chronic inflammation or infection) may also have higher levels of oxidative stress.

\section{Infection}

In their series, Kane et al found that patients who developed methemoglobinemia were more likely to have active systemic infection than controls $(68.4 \%$ vs $6.8 \% ; P<0.001){ }^{2}$ Systemic infection is a powerful cause of oxidative stress which in turn increases methemoglobin production. Methemoglobin levels are increased in sepsis due to the excess production of nitric oxide. ${ }^{19}$ The susceptibility of patients with sepsis to methemoglobinemia appears to be 
due to a combination of factors in addition to the infectious process itself. Metabolism of anesthetics and elimination of oxidizing metabolites may be altered in patients who are seriously ill from infectious processes. In addition, patients with infections are more likely to be exposed to drugs such as dapsone, sulfonamides, and acetaminophen. In our experience with 886 patients undergoing TEE, patients who had an active systemic infection were more likely to have lower hemoglobin and albumin levels and higher use of acetaminophen and sulfa drugs $(P<0.001){ }^{4}$

\section{Hypoalbuminemia}

The presence of low albumin may lead to increased concentration of free benzocaine molecules which in turn cause methemoglobin production. ${ }^{20}$

\section{Hypoxia and pre-existing lung disease}

Presence of hypoxia may provoke symptoms of methemoglobinemia at a lower methemoglobin level.

\section{Pre-existing liver and cardiac disease}

Amide anesthetics are predominantly metabolized in the liver via the cytochrome P450 system. ${ }^{12}$ Pre-existing liver disease may impair drug metabolism. Cardiac dysfunction may cause decreased perfusion of the liver thereby decreasing hepatic function and altering metabolism. This may not be a major concern with metabolism of ester anesthetics, but may contribute to the overall state of increased oxidative stress.

\section{Drugs}

Numerous drugs have been implicated as risk factors for the development of methemoglobinemia and are enumerated in Table $1 .{ }^{18,21}$ When patients who take these medications are exposed to benzocaine, the production of methemoglobin may overwhelm the body's reductive capacity.

Table I List of medications commonly used in clinical practice that have been linked to methemoglobinemia ${ }^{17,20}$

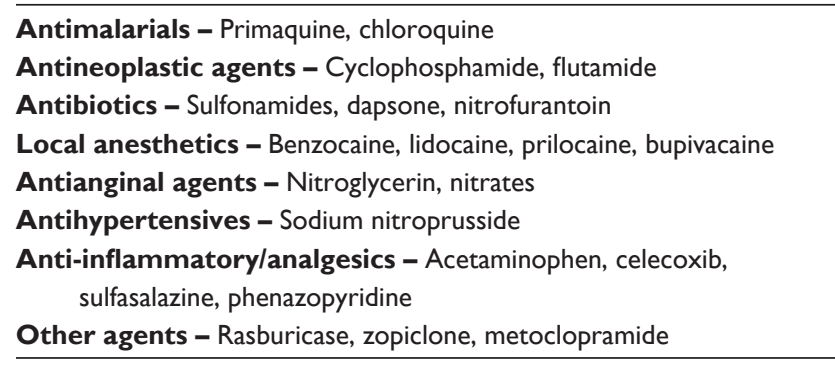

\section{Dietary factors}

The influence of diet on occurrence of methemoglobinemia in the setting of endoscopy has not been well studied. Nitrites and nitrates are commonly used in processed food as a preservative. ${ }^{17,22}$ Higher concentrations of nitrites in well water have caused clusters of acute methemoglobinemia both in the United States and other countries. Patients who are exposed to such oxidative agents through their diet may constitute a high risk group when exposed to benzocaine.

\section{Hospitalization}

Hospitalized patients who undergo TEE are at a higher risk of developing methemoglobinemia. ${ }^{2}$ Patients who are hospitalized are likely to have a number of the above comorbidities and contributing factors such as infection and increased use of drugs. The alteration of gut flora in hospitalized patients as an inciting factor has not been studied. In infants, the low stomach acid and overgrowth of nitrite-reducing bacteria due to intestinal $\mathrm{pH}$ changes predispose to methemoglobin production. ${ }^{18}$ Similar changes may occur in critically ill adult patients who are concomitantly treated with proton-pump inhibitors.

\section{Genetic factors}

Differences in metabolism of anesthetics may account for increased production of toxic metabolites as described above. In addition, the genetic abnormalities in the reductive pathways may also influence risk. There are two forms of autosomal recessive deficiency of cytb5R. The deficiency in type 1 is limited to the red blood cells. Homozygous individuals present with cyanosis at birth and are readily identified. Heterozygotes are often asymptomatic until challenged by toxins or drugs. Type 2 deficiency occurs in all cells and is associated with mental retardation. Hemoglobin $M$ is an autosomal dominant condition where the replacement of a histidine residue by tyrosine permits oxidation of iron. Patients with hemoglobin $\mathrm{M}$ present with cyanosis.

Of all these genetic factors, the most likely scenario in the setting of endoscopy is when a previously undiagnosed type 1 heterozygote is exposed to a topical anesthetic. However, in the single study done to date, most patients who develop methemoglobinemia due to topical anesthetics do not have detectable genetic deficiency. ${ }^{23}$ This is also supported by the observation that the risk of methemoglobinemia in the same individual may increase with changes in the body's milieu. A case report by Udeh et al illustrates how increased oxidative stress causes methemoglobinemia with benzocaine even when a prior exposure was uneventful. ${ }^{20} \mathrm{~A}$ critically ill patient is described who had an initial uneventful exposure to 
$14 \%$ benzocaine but developed methemoglobinemia with a $20 \%$ benzocaine spray 3 days later in the setting of worsening sepsis, hypoalbuminemia, and acetaminophen use.

\section{Procedure-specific factors}

There are no studies which directly compared the incidence of methemoglobinemia during various endoscopic procedures. However, in the case report literature most cases occur in the setting of transesophageal echocardiography. Fewer cases have been reported with esophagoscopy or bronchoscopy. In spite of the possibility of obvious bias, it is reasonable to hypothesize that TEE may be a predisposing factor. TEE is often performed without visual fiber optic guidance. It is not unreasonable to assume that more mucosal trauma would occur with a blind procedure rather than one where the tip of the tube is guided visually. Such trauma may cause increased absorption of the anesthetic. The clinical setting in which these procedures occur is also important. For example, a TEE performed to rule out vegetation in a septic patient may be more likely venue for occurrence of methemoglobinemia than a screening esophagoscopy for Barrett's esophagus in an asymptomatic patient. More research is needed to confirm this hypothesis.

\section{Clinical manifestations}

The development of clinical manifestations depends on the level of methemoglobin, baseline hemoglobin, and the presence of comorbidities such as heart and lung disease which may predispose patients to symptoms at lower levels. These manifestations and the level at which they are seen is shown in Table $2 .{ }^{21}$

\section{Diagnosis}

The disease should be suspected in all patients who develop hypoxia, cyanosis, or tachypnea in the setting of endoscopy. Pulse oximetry which is commonly used to monitor patients using endoscopy is not useful in the diagnosis of methemoglobinemia. With higher levels of methemoglobin,

Table 2 Clinical symptoms and signs of methemoglobinemia in relation to the level of methemoglobin

\begin{tabular}{ll}
\hline Level of methemoglobin & Clinical symptoms and signs \\
\hline$<10 \%$ & Frequently asymptomatic, \\
& occasionally grayish skin \\
$10 \%-20 \%$ & Skin changes such as cyanosis \\
$20 \%-30 \%$ & Dyspnea, headache, anxiety \\
$30 \%-50 \%$ & Dizziness, palpitations, \\
& confusion, tachypnea \\
$50 \%-70 \%$ & Seizures, cardiac arrhythmias, \\
& metabolic acidosis, coma \\
$>70 \%$ & Death \\
\hline
\end{tabular}

the patient's blood acquires a unique chocolate brown color. Measurement of methemoglobin levels in arterial blood by co-oximetry is diagnostic. Noninvasive co-oximetry devices are now available, ${ }^{17}$ which can be used to monitor levels in real time.

\section{Treatment}

Treatment consists of general supportive care and administration of intravenous methylene blue usually at a dose of $1-2 \mathrm{mg} / \mathrm{kg}$ over 5 minutes. Methylene blue through its metabolite leukomethylene blue (reducing agent) helps activate the flavin-NADPH pathway which is normally quiescent. It is recommended in symptomatic patients with a methemoglobin level more than $20 \%$ and in asymptomatic patients with a level above $30 \%{ }^{24}$ Treatment should also be considered at lower doses in patients with serious co-morbidities (such as heart and lung disease) which make them more susceptible to the effects of methemoglobinemia. Methylene blue may not effective in patients with glucose 6 dehydrogenase deficiency because insufficient NADPH is produced in this condition. It should be dosed carefully since excessive doses can cause hemolysis and paradoxical methemoglobinemia (since there is excess methylene blue compared to leukomethylene blue). An infusion of dextrose can be considered since it is necessary for the production of NADH and NADPH which are essential for the reductive pathways. ${ }^{18}$ Doses of methylene blue can be repeated up to a maximum total dose of $7 \mathrm{mg} / \mathrm{kg}$. Exchange transfusion is used in severe cases where methylene blue therapy has been ineffective. Patients should be carefully monitored for at least 24 hours since some may develop rebound methemoglobinemia.

\section{Areas of uncertainty}

Our knowledge of topical anesthetic-induced methemoglobinemia is severely limited by the lack of rigorously designed prospective studies. Most of our current understanding is from case reports and retrospective studies. The actual dose of anesthetic administered is often not clear. Multiple anesthetics may be concomitantly used, so attributing blame to a single drug is not possible. There may be several patient factors that are missed in a retrospective review of records. The other important limitation is the fact that many minimally symptomatic cases are probably not diagnosed.

\section{Suggestions for design of future studies}

A carefully designed prospective study is needed to test the findings of available reports which should only be considered 
hypothesis generating. This future study should randomize patients undergoing endoscopy to benzocaine and lidocaine. The study should also attempt to test the dose-response relationship in various subsets of patients based on their predisposing factors and in different endoscopy settings. Demographic data collected should include baseline medication use, dietary nitrite intake, and other comorbid conditions. Baseline methemoglobin levels should be obtained. The dose of anesthetic administered should be clearly documented. Continuous methemoglobin monitoring would be preferred to random sampling. Noninvasive determination of methemoglobin levels is now possible and is preferred to invasive collection of arterial blood. The knowledge gained from such a study would be immensely helpful in furthering our knowledge of methemoglobinemia.

\section{Conclusion}

The occurrence of acquired methemoglobinemia due to topical anesthetics appears to be the consequence of a "perfect storm" of predisposing anesthetic, patient, and possibly procedure-related factors. Since most cases of benzocaineassociated methemoglobinemia occurred in patients with readily identifiable risk factors (infection, anemia, etc), it may be prudent to avoid benzocaine in this population. Prospective studies using continuous measurement of methemoglobin levels are needed to better understand this problem and to develop recommendations to prevent it.

\section{Disclosure}

The author reports no conflicts of interest in this work.

\section{References}

1. Umbreit J. Methemoglobin - it's not just blue: a concise review. Am J Hematol. 2007;82(2):134-144.

2. Kane GC, Hoehn SM, Behrenbeck TR, Mulvagh SL. Benzocaine-induced methemoglobinemia based on the Mayo Clinic experience from 28,478 transesophageal echocardiograms: incidence, outcomes, and predisposing factors. Arch Intern Med. 2007;167(18):1977-1982.

3. Novaro GM, Aronow HD, Militello MA, Garcia MJ, Sabik EM. Benzocaine-induced methemoglobinemia: experience from a highvolume transesophageal echocardiography laboratory. J Am Soc Echocardiogr. 2003;16(2):170-175.

4. Vallurupalli S, Das S, Manchanda S. Infection and the risk of topical anesthetic induced clinically significant methemoglobinemia after transesophageal echocardiography. Echocardiography. 2010;27(3):318-323.
5. Douglas WW, Fairbanks VF. Methemoglobinemia induced by a topical anesthetic spray (Cetacaine). Chest. 1977;71(5):587-591.

6. Ash-Bernal R, Wise R, Wright SM. Acquired methemoglobinemia: a retrospective series of 138 cases at two teaching hospitals. Medicine (Baltimore). 2004;83(5):265-273.

7. Moore TJ, Walsh CS, Cohen MR. Reported adverse event cases of methemoglobinemia associated with benzocaine products. Arch Intern Med. 2004;164(11):1192-1196.

8. Guay J. Methemoglobinemia related to local anesthetics: a summary of 242 episodes. Anesth Analg. 2009;108(3):837-845.

9. Moos DD, Cuddeford JD. Methemoglobinemia and benzocaine. Gastroenterol Nurs. 2007;30(5):342-345.

10. Prchal JT. Genetics and pathogenesis of methemoglobinemia. In: Basow DS. editor. Up To Date. Waltham, MA; 2010.

11. Kinoshita A, Nakayama Y, Kitayama T, Tomita M. Simulation study of methemoglobin reduction in erythrocytes. Differential contributions of two pathways to tolerance to oxidative stress. FEBS J. 2007;274(6):1449-1458.

12. Catterall WA, Mackie K. Local anesthetics. In: Brunton LL, Lazo JS, Parker KL (editors). Goodman and Gilman's The Pharmacological Basis of Therapeutics, 2005;11e. Available from: http://www. accessanesthesiology.com/content/938035. Accessed Oct 12010 .

13. Guertler AT, Lagutchik MS, Martin DG. Topical anesthetic-induced methemoglobinemia in sheep: a comparison of benzocaine and lidocaine. Fundam Appl Toxicol. 1992;18(2):294-298.

14. Guertler AT, Pearce WA. A prospective evaluation of benzocaineassociated methemoglobinemia in human beings. Ann Emerg Med. 1994;24(4):626-630.

15. Gupta PM, Lala DS, Arsura EL. Benzocaine-induced methemoglobinemia. South Med J. 2000;93(1):83-86.

16. Khorasani A, Candido KD, Ghaleb AH, Saatee S, Appavu SK. Canister tip orientation and residual volume have significant impact on the dose of benzocaine delivered by Hurricaine spray. Anesth Analg. 2001;92(2):379-383.

17. do Nascimento TS, Pereira RO, de Mello HL, Costa J. [Methemoglobinemia: from diagnosis to treatment]. Rev Bras Anestesiol. 2008;58(6): 651-664.

18. Rehman HU. Methemoglobinemia. West J Med. 2001;175(3):193-196.

19. Ohashi K, Yukioka H, Hayashi M, Asada A. Elevated methemoglobin in patients with sepsis. Acta Anaesthesiol Scand. 1998;42(6):713-716.

20. Udeh C, Bittikofer J, Sum-Ping ST. Severe methemoglobinemia on reexposure to benzocaine. J Clin Anesth. 2001;13(2):128-130.

21. Denshaw-Burke M, Curran AL. Methemoglobinemia. 2010 May 3. Available from: http://emedicine.medscape.com/article/204178-overview. Accessed Nov 52010.

22. Greer FR, Shannon M; American Academy of Pediatrics Committee on Nutrition; American Academy of Pediatrics Committee on Environmental Health. Infant methemoglobinemia: the role of dietary nitrate in food and water. Pediatrics. 2005;116(3):784-786.

23. Maran J, Guan Y, Ou CN, Prchal JT. Heterogeneity of the molecular biology of methemoglobinemia: a study of eight consecutive patients. Haematologica. 2005;90(5):687-689.

24. Price D. Methemoglobinemia. In: Goldfrank LR, Flomenbaum NE, Lewin NA, Weisman RS, Howland MA, Hoffman RS. editors. Goldfrank's Toxicologic Emergencies. 6th ed. Old Tappan, NJ: Appleton and Lange; 1998:1507-1523.

\section{Publish your work in this journal}

Local and Regional Anesthesia is an international, peer-reviewed, open access journal publishing on the development, pharmacology, delivery and targeting and clinical use of local and regional anesthetics and analgesics. The journal welcomes submitted papers covering original research, basic science, clinical studies, reviews \& evaluations,

guidelines, expert opinion and commentary, case reports and extended reports. The manuscript management system is completely online and includes a very quick and fair peer-review system, which is all easy to use. Visit http://www.dovepress.com/testimonials.php to read real quotes from published authors.

\section{Dovepress}

Submit your manuscript here: http://www.dovepress.com/local-and-regional-anesthesia-journal 